\title{
Medium-chain fatty acids accelerate the incorporation of eicosapentaenoic acid into lung tissue
}

Christopher Beermann ( $\nabla$ christopher.beermann@lt.hs-fulda.de)

University of Applied Sciences Fulda: Hochschule Fulda

Daniela Fussbroich

University of Applied Sciences Fulda: Hochschule Fulda

Ralf Schubert

University of Frankfurt: Goethe-Universitat Frankfurt am Main

\section{Short report}

Keywords: asthma, fat metabolic rate, immune response, long-chain polyunsaturated fatty acids, medium-chain fatty acids, resolving inflammation, resorption kinetics

Posted Date: February 7th, 2021

DOI: https://doi.org/10.21203/rs.3.rs-120503/v2

License: (c) (1) This work is licensed under a Creative Commons Attribution 4.0 International License. Read Full License 
1 Medium-chain fatty acids accelerate the incorporation of eicosapentaenoic acid into

2 lung tissue

3 Christopher Beermann (corresponding author)

4 Department of Food Technology, University of Applied Science Fulda, Leipziger Str. 123, 536039 Fulda, Germany.

6 christopher.beermann@It.hs-fulda.de

7 Daniela Fussbroich

8 Department of Food Technology, University of Applied Science Fulda, Leipziger Str. 123, 936039 Fulda, Germany.

10 Ralf Schubert

11 Division for Allergy, Pneumology and Cystic Fibrosis, Department for Children and 12 Adolescence, Goethe University, Theodor-Stern-Kai 7, Frankfurt/Main, Germany. 


\section{Abstract}

Long-chain polyunsaturated fatty acids (LCPUFAs) are reported to resolve chronic inflammation in asthma and other lung diseases. This study aimed to accelerate the incorporation of eicosapentaenoic acid (EPA) into lung tissue through the coapplication of medium-chain fatty acids (MCFAs) which enhance the fat-metabolic rate.

Female C57BL/6 mice were supplemented with either 1363,6 mg EPA or 1363,6 mg EPA and MCFAs at $30 \%$ of the total fat per kg body weight per day for 28 days (each group size: $n=21$ ). The resorption of EPA into the peripheral blood and lung tissue was monitored over 63 days including the wash-out phase.

In the peripheral blood plasma and clots the supplementation with EPA always led to higher EPA concentrations than the administration of EPA with MCFAs pointing to a preferred EPA incorporation into tissues induced by MCFAs (EPA in plasma at day 26: EPA 12.33 wt\% \pm 1.41; EPA and MCFAs $3.91 \mathrm{wt} \% \pm 0.32 ; \Delta 8.42 ; p<0.001$; EPA in clots at day 26: EPA 16.44 $w t \% \pm 1.82$; EPA and MCFAs $4.47 w t \% \pm 1.26 ; \Delta 11.97 ; p<0.001)$. In the lung tissue the EPAincorporation at day 26 was increased by MCFAs compared to the EPA-administration alone (EPA in lung tissue at day 26: EPA $1.28 w t \% \pm 0.18$; EPA and MCFAs $1.83 w t \% \pm 0.17 ; \Delta$ $0.55 ; p<0.01)$.

The present study recommends the use of dietary LCPUFA supplementation with MCFAs to support their incorporation into lung tissues.

\section{Keywords}

asthma, fat metabolic rate, immune response, long-chain polyunsaturated fatty acids, mediumchain fatty acids, resolving inflammation, resorption kinetics

\section{Abbreviations}

EPA: eicosapentaenoic acid

DHA: docosahexaenoic acid

MCFAs: medium-chain fatty acids

LCPUFAs: polyunsaturated fatty acids

\section{Acknowledgment}


45 The authors would like to thank Annekathrin Goepel for excellent technical help and are 46 grateful for the supportive PhD-grant of Cusanuswerk, Germany.

\section{$47 \quad$ Funding}

$48 \quad$ Not applicable.

49 Declaration of Competing Interest

50 The authors declare that they have no known competing financial interests or personal

51 relationships that could have appeared to influence the work reported in this paper

\section{Ethics Statements File}

53 All animal procedures were performed according to protocols approved by the German Animal 54 Subjects Committee (Gen.Nr.FK/1036).

55 Competing interests

56 The authors declare that they have no competing interests. 


\section{Introduction}

Polyunsaturated fatty acids (LCPUFAs) are essential components of cellular membranes that are important for maintaining the structure and function of cells, tissues and organs. In addition to treating heart diseases or mental illnesses, dietary LCPUFA supplementation has been discussed as a treatment that is used to reverse fatty acid profile alterations and to resolve chronic inflammation in asthma and other lung diseases in different experimental models (Levi \& Serhan, 2014). Bronchial allergic asthma, for instance, is characterized by dysregulated immune reactions, with the hyperinflammation of the lung bronchi and, in the late phase, lung tissue infiltration by eosinophilic granulocytes, provoking typical symptoms (Schubert et al., 2009). LCPUFAs are metabolic precursors of several lipid-mediators regulating the initiation, the containment and resolution of inflammation (Beermann, Neumann, Schubert \& Zielen, 2016). Due to ongoing inflammation, a lack of immune-regulative LCPUFAs can occur in the cellular membranes of lung tissue and immune cells which should be retreated (Fussbroich et al., 2019). The absorption and incorporation process into tissues of dietary LCPUFAs is metabolically complex and time consuming. To establish effective LCPUFA-supplementation strategies against immunological hyperreactions in asthma and other lung diseases, this study aimed to accelerate the incorporation of orally applied LCPUFAs into lung tissue by coapplying medium-chain fatty acids (MCFAs, C6:0 - C12:0). This study is part of a mouse trial focusing on the immunological and pathophysiological aspects of LCPUFA supplementation in asthma (Fussbroich et al., 2020).

\section{Material and methods}

Mice keeping

Female C57BL/6 mice (Charles River Laboratories, Wilmington, Delaware) at the age of 6-8 weeks were maintained in stainless steel cages under sterile conditions with an alternating 12 h light/dark cycle. Mice were fed water and laboratory chow (Ssniff, Soest, Germany) with 6\% fat content (C 14:0: 0.01\%; C 16:0: 0.68\%; C 16:1:0.04\%; C 18:0: 0.22\%; C 18:1: 1.44\%; C 18:2: 3.21\%; C 18:3: 0.37\%; C20:0: 0.03\%; C 20:1: 0.01\%) ad libitum. The body weight of the mice was documented twice per week. All animal procedures were performed according to protocols approved by the German Animal Subjects Committee (Gen.Nr.FK/1036).

Study design and dietary supplementation

Mice were randomized into two dietary treatment groups, eicosapentaenoic acid, (EPA C20:5 n3) $(n=21)$ and EPA + MCFAs ( $n=21)$ groups, as well as a control group $(n=3)$. Each treatment group was fed the experimental diet for 28 days (wash-in period); afterwards, the wash-out period without any supplementation was observed until day 63 . 
An experimental diet containing $1363,6 \mathrm{mg}$ EPA or $1363,6 \mathrm{mg}$ EPA plus MCFAs at $30 \%$ of the total fat per kg body weight per day was applied orally to each mouse daily from day 1 up to day 28. The experimental diet was applied orally through a feeding needle (canula, $0.70 \times 30$ $\mathrm{mm}$, LL, curved, Knopf C, Robert Helwig GmbH, Berlin, Germany) connected to a $1 \mathrm{~mL}$ syringe (Becton Dickinson, Frankfurt/Main, Germany). During supplementation, the experimental diet was freshly mixed on a daily basis from commercially available dietary EPA and MCFA oil and diluted in $0.5 \%$ (w:v) gum arabic solution (gum arabic powder, Carl Roth, Karlsruhe, Germany; sterile water) to a final volume of $200 \mu \mathrm{L}$ per dose to ensure that a defined and precise application volume was given. Just before administration, emulsions were homogenized using an ultrasonic homogenizer (Sonopuls, Bandelin, Berlin, Germany).

Blood and tissue collection

Blood was collected on day 2 and then every second day until day 26 by venipuncture of the vena facialis. On days $0,28,31,35,42,49,56$ and 63 mice were sacrificed by cardiac withdrawal under anesthetic. All blood samples were collected in EDTA-microvettes (Sarstedt, Nümbrecht, Germany). Whole blood was centrifuged by using a Biofuge-Fresco (Heraeus, Hanau, Germany) at $10000 \mathrm{rpm}$ for 10 minutes, and the remaining cruor was stored in collection tubes at $-80^{\circ} \mathrm{C}$ until further measurement. Lungs were flushed with PBS through the pulmonary artery to wash out erythrocytes. After this, the lungs were removed and stored at $80^{\circ} \mathrm{C}$ until further measurement.

Analysis of the fatty acid profile

Total lipids of lung tissue, blood clots and plasma were extracted according to the method of Bligh \& Dyer (Bligh \& Dyer, 1959). For the lipid extraction, the lung tissue and blood clots were lysed and homogenized with an ammonium chloride potassium lysis buffer (Sigma-Aldrich, Taufkirchen, Germany). The total lipid fraction from the extractions was completely dried using a CentriVap (Jouan RC1010, Thermo Scientific, Milan, Italy), overlaid with nitrogen, and then derivatized to fatty acid methyl esters according to the method of Kohn (Kohn, van der Ploeg, Möbius, \& Sawatzki, 1996). After derivatization, the organic solvent was completely dried and the fatty acid methyl esters were resolved in hexane (Sigma-Aldrich, Taufkirchen, Germany) for measurement by capillary gas chromatography flame-ionization detection (Fussbroich et al., 2020).

Statistics

Data are displayed as the mean \pm standard error of the mean, and were analyzed using a twoway ANOVA with Bonferroni post hoc analysis using GraphPad Prism 5 (GraphPad Software, La Jolla, CA). Differences were considered statistically significant at $p<0.05$. 
127 Fatty acids undergo different metabolic fates depending on their chain length and degree of saturation. Ingested LCPUFAs are absorbed in the small intestine, and re-esterified in enterocytes to newly formed triglycerides or phospholipids to be subsequently packaged into chylomicrons within hours in the postprandial phase (Green \& Riley, 1981; Mu et al., 2006). These LCPUFA-loaded lipoproteins are secreted to the lymph and afterwards to the bloodstream through the thoracic duct. At least in the peripheral tissues and organs, LCPUFAs are released by lipoprotein lipases and are transported into the cells to undergo different metabolic fates, including membrane and lipid mediator synthesis, as well as oxidation in mitochondria or esterification and storage in lipid droplets (Roche \& Gibney, 1999). Depending on the applied amount, concise LCPUFA incorporation into cellular membranes of erythrocytes for up to 28 days is needed in humans, and into organ tissues for even more time (LopezMiranda, Williams \& Lairon, 2007). In this study with mice, an EPA supplementation period of 28 days was established followed by a 35-day wash-out phase without any supplementation.

To improve the effectiveness of dietary LCPUFA supplementation, MCFAs might be helpful to accelerate tissue incorporation. Generally, short carboxylic acids with 6- to 12-carbon-long aliphatic chains are derived from palm kernel or coconut oil, as well as from milk fat in the form of triglycerides. They are used in low-carbohydrate, high-fat ketogenic diets for the treatment of epilepsy, for fat tissue reduction against obesity, or in athletic preparations (Levy, Cooper, Giri \& Weston, 2012). In contrast to LCPUFAs, the resorption of consumed MCFAs begins by the oral and gastric mucosa and is ultimately ingested by the small intestine. Then MCFAs are transported in the portal blood system, associated with serum albumin, directly to the liver. Independent of the carnitine acyltransferase transport system, MCFAs immediately undergo mitochondrial oxidation. In different animal models and in obese subjects, MCFA supplementation increases postprandial energy expenditure, thermogenesis and attenuated weight accretion or weight loss (Rego Costa, Rosado \& Soares-Mota, 2012). In another clinical trial, dietary supplementation of healthy volunteers with MCFAs and LCPUFAs led to increased blood fat clearance and fat oxidation (Beermann et al., 2003). Fat metabolism stimulated by MCFAs with increased fatty acid resorption together with preferred mitochondrial MCFA oxidation competitive with LCPUFAs might metabolically direct ingested LCPUFAs away from catabolic digestion and towards tissue incorporation.

The incorporation kinetics of LCPUFAs into cellular membranes diverge depending on the LCPUFA species, the amount of application, and the type of supplemented cellular membrane system. In particular, the incorporation of EPA and docosahexaenoic acid (DHA, 22:6 n3) into cellular membranes is different depending on the type of cell. Moreover, studies with rats have demonstrated that the incorporation of EPA into peripheral and organ tissues is lower than that 
of DHA (Owen et al., 2004). Therefore, in the present study, EPA was used as a lowincorporation resorption marker for LCPUFAs. Applying EPA alone resulted in an increased concentration of this fatty acid in the peripheral blood plasma, reaching a plateau at day 10 up to day 28 of supplementation (Fig. 1 A). In contrast, a combined administration of EPA with MCFAs never reached this concentration level (EPA in plasma at day 26: EPA $12.33 \mathrm{wt} \% \pm$ 1.41; EPA and MCFAs 3.91 wt $\% \pm 0.32 ; \Delta 8.42 ; p<0.001$ ). After day 28 , during the wash-out phase, the EPA concentration rapidly decreased in plasma. A comparable outcome was evident for peripheral blood clots (Fig. 1 B). Generally, erythrocytes are a good indicator of LCPUFA incorporation into cellular membranes in stable diets. However, they vary more in the pattern of incorporation compared to organ tissues during the LCPUFA-supplementation period (Owen et al., 2004). One factor for this might be the shorter life span of erythrocytes, at 40 days in mice, than of lung tissue cells (Horký, Vácha, \& Znojil, 1978). In this study, supplementing EPA alone led to an increased concentration of this fatty acid in the peripheral blood clots, reaching an unsteady concentration plateau at day 10 up to day 26 of supplementation, which was always higher than the combined administration of EPA with MCFAs pointing to a preferred internal incorporation of EPA into cellular membranes of organ tissues induced by MCFAs (EPA in clots at day 26: EPA $16.44 \mathrm{wt} \% \pm 1.82$; EPA and MCFAs 4.47 wt $\% \pm 1.26 ; \Delta 11.97 ; p<0.001$; Fig. 1 B). Indeed, MCFAs significantly increased the EPA incorporation into the bronchial tissue of the lung up to day 26 by more than a one-third compared to EPA administration alone (EPA in lung tissue at day 26: EPA $1.28 \mathrm{wt} \% \pm 0.18$; EPA and MCFAs $1.83 w t \% \pm 0.17 ; \Delta 0.55 ; p<0.01$; Fig. 2). Due to the preferred mitochondrial MCFA oxidation, the supplemented EPA seem to be spared from catabolic processes and preferentially shifted towards incorporation into cellular membranes of the lung tissue. The body weights of both experimental groups never altered in this setting. In contrast to our findings, in rats faltering LCPUFA-contents have already been observed after 2 days of supplementation in different organs, such as in the myocardium and skeletal muscle. Furthermore, stable maximal incorporation occurred after 28 days of supplementation (Owen et al., 2004). In this study, a stable saturated level of EPA could never be reached in the lung tissue within 28 days of supplementation, which may be due to the different basal metabolic rates of mice compared to rats. Interestingly, the removal of cellular-associated EPA was also enhanced by MCFAs beyond the supplementation phase compared to EPA alone, indicating that the stimulated rate of fat metabolism continues beyond supplementation (EPA in lung tissue at day 31: EPA $0.81 w t \% \pm 0.08$; EPA and MCFAs $0.58 w t \% \pm 0.06 ; \Delta 0.23 ; p<0.01$; Fig. 2). This finding has to be considered in the supplementation strategy for immune-regulative LCPUFA supplementation against lung inflammation.

\section{Conclusions}


198 Based on the illustrated accelerating effects of MCFAs on the incorporation and elimination 199 kinetics of dietary LCPUFAs into and from lung tissue, the present study suggests to 200 accompany dietary supplementation with both LCPUFAs and MCFA to support LCPUFA 201 incorporation into lung tissue. Upon the termination of supplementation, MCFAs should be 202 discontinued to stabilize the established LCPUFA status in the body. 
204

Levy, B. D., \& Serhan, C. N. (2014). Resolution of Acute Inflammation in the Lung. Annual Review of Physiology, 76, 467-492. https://doi.org/10.1146/annurev-physiol-021113-170408.

Schubert, R., Kitz, R., C., Beermann, C., Rose, M. A., Lieb, A., P.C. Sommerer, P. C., Moskovits, J., Alberternst, H., Böhles, H. J., J. Schulze, J., \& Zielen, S. (2009). Effect of n-3 polyunsaturated fatty acids in asthma after low-dose allergen challenge. International Archive of Allergy and Immunology, 48, 321-329. https://doi.org/148:321-329.10.1159/000170386.

Beermann, C., Neumann, S., Schubert, R., \& Zielen, S. (2016). Combinations of distinct longchain polyunsaturated fatty acid species for improved dietary treatment against allergic bronchial asthma. Nutrition, 32. https://doi.org/11-12.1165-70.10.1016/j.nut.2016.04.004.2.

Fussbroich, D., Zimmermann, K., Göpel, A., Eickmeier, O., Trischler, J., Zielen, S., Schubert R., \& Beermann C. (2019). A specific combined long-chain polyunsaturated fatty acid supplementation reverses fatty acid profile alterations in a mouse model of chronic asthma. Lipids in Health and Disease,18, 16. https://doi.org/10.1186/s12944-018-0947-6.

Fussbroich, D., Colas, R. A., Eickmeier, O., Trischler, J., Jerkic, S. P., Zimmermann, K., Göpel, A., Schwenger, T., Schaible, A., Henrich, D., Baer, P., Zielen, S., Dalli, J., Beermann, C., \& Schubert, R. (2020). A combination of LCLCPUFA ameliorates airway inflammation in asthmatic mice by promoting pro-resolving effects and reducing adverse effects of EPA. Mucosal Immunololgy, 13, 1-12. https://doi.org/10.1038/s41385-019-0245-2.

Bligh, E. G., \& Dyer, W. J. (1959). A rapid method of total lipid extraction and purification. Canadian Journal of Biochemistry and Physiology, 37, 8, 911-7. https://doi.org/10.1139/o59$\underline{099}$.

Kohn, G., van der Ploeg, P., Möbius, M., \& Sawatzki, G. (1996). Influence of the derivatization procedure on the results of the gaschromatographic fatty acid analysis of human milk and infant formulae. Zeitschrift für Ernährungswissenschaften. 35, 3, 226-34. 10.1 https://doi.org/007/BF01625685.

Green, P. H., \& Riley, J. W. (1981). Lipid absorption and intestinal lipoprotein formation. Australien and New Zealand Journal of Medicine, 11, 1, 84-90. https://doi.org/10.1111/j.14455994.1981.tb03746.x.

Mu, H., Thøgersen, R. L., Maaetoft-Udsen, K., Straarup, E. M., \& Frøkiaer, H. (2006). Different kinetic in incorporation and depletion of $n-3$ fatty acids in erythrocytes and leukocytes of mice. Lipids, 41, 8, 749-52. https://doi.org/10.1007/s11745-006-5026-5. 
235 Roche, H. M., \& Gibney, M. J. (1999). Long-chain n-3 polyunsaturated fatty acids and

236 triacylglycerol metabolism in the Postprandial State. Lipids, 34, S259-S265.

237 https://doi.org/10.1007/BF02562313.

238 Lopez-Miranda, J., Williams, C., \& Lairon, D. (2007). Dietary, physiological, genetic and

239 pathological influences on postprandial lipid metabolism. British Journal of Nutrition, 98, 458-

240 473. https://doi.org/10.1017/S000711450774268X.

241 Levy, R. G., Cooper, P. N., Giri, P., \& Weston, J. (2012). Ketogenic diet and other dietary

242 treatments for epilepsy. Cochrane Database of Systematic Reviews 3:CD001903.

243 https://doi.org/10.1002/14651858.CD001903.pub2.

244 Rego Costa, A.C., Rosado, E.L., \& Soares-Mota, M. (2012). Influence of the dietary intake of 245 medium chain triglycerides on body composition, energy expenditure and satiety; a systematic 246 review. Nutrition Hospitalaria, 227, 103-108. https://doi.org/10.3305/nh.2012.27.1.5369.

247 Beermann, C., Jelinek, J., Reinecker, T., Hauenschild, A., Boehm, G., \& Klör, H. U. (2003). 248 Short term effects of dietary medium-chain fatty acids and n-3 long-chain polyunsaturated fatty 249 acids on the fat metabolism of healthy volunteers. Lipids in Health and Disease, 2, 1. 250 https://doi.org/10.1186/1476-511X-2-10.

251 Owen, A. J. \& Peter-Przyborowska, B. A., Hoy A. J., \& McLennan, P. L. (2004). Dietary fish oil 252 dose- and time-response effects on cardiac phospholipid fatty acid composition. Lipids, 39, 10, 253 955-61. https://doi.org/10.1007/s11745-004-1317-0.

254 Horký, J., Vácha, J., \& Znojil, V. (1978). Comparison of life span of erythrocytes in some inbred 255 strains of mouse using 14C-labelled glycine. Physiologia Bohemoslovaca, 27, 3, 209-17. 


\section{Legend}

258 Fig. 1. Concentration of EPA in the peripheral blood plasma (A) and clots (B) during and 259 after supplementation. Female C57BL/6 mice were supplemented with 1363,6 mg EPA 260 (group size: $n=21$ ) or 1363,6 EPA and MCFAs at $30 \%$ of the total fat per kg body weight per day (group size: $n=16$ ) for 28 days. ${ }^{* *} p<0.01,{ }^{* * *} p<0.001,{ }^{\# \# \#} P<0.01$ compared to baseline.

262 In the peripheral blood plasma and clots the supplementation with EPA increased its concentration always higher compared to the combined administration of EPA with MCFAs pointing to a preferred EPA incorporation into tissues induced by MCFAs (EPA in plasma at day 26: EPA $12.33 w t \% \pm 1.41$; EPA and MCFAs $3.91 w t \% \pm 0.32 ; \Delta 8.42 ; p<0.001$; EPA in clots at day 26: EPA $16.44 \mathrm{wt} \% \pm 1.82 ;$ EPA and MCFAs $4.47 \mathrm{wt} \% \pm 1.26 ; \Delta 11.97 ; p<0.001)$.

Fig. 2. Concentration of EPA in lung tissue along and after supplementation. Female C57BL/6 mice were supplemented with $1000 \mathrm{mg}$ EPA (group size: $\mathrm{n}=21$ ) or 1363,6 mg EPA (group size: $n=21$ ) or 1363,6 EPA and MCFAs at $30 \%$ of the total fat per kg body weight per day (group size: $n=21$ ) for 28 days. ${ }^{* * *} p<0.01$ between the groups, ${ }^{\# \#} p<0.01$ compared to baseline.

273 The combined administration of EPA and MCFAs increased EPA incorporation into the lung 274 tissue up to day 26 (EPA in lung tissue at day 26: EPA $1.28 \mathrm{wt} \% \pm 0.18$; EPA and MCFAs 1.83 $275 w t \% \pm 0.17 ; \Delta 0.55 ; p<0.01)$ and disappeared earlier thereon compared to EPA supplementation alone (EPA in lung tissue at day 31: EPA $0.81 \mathrm{wt} \% \pm 0.08$; EPA and MCFA 0.58 wt $\% \pm 0.06 ; \Delta 0.23 ; p<0.01)$. 
Figure 1. EPA concentration in blood
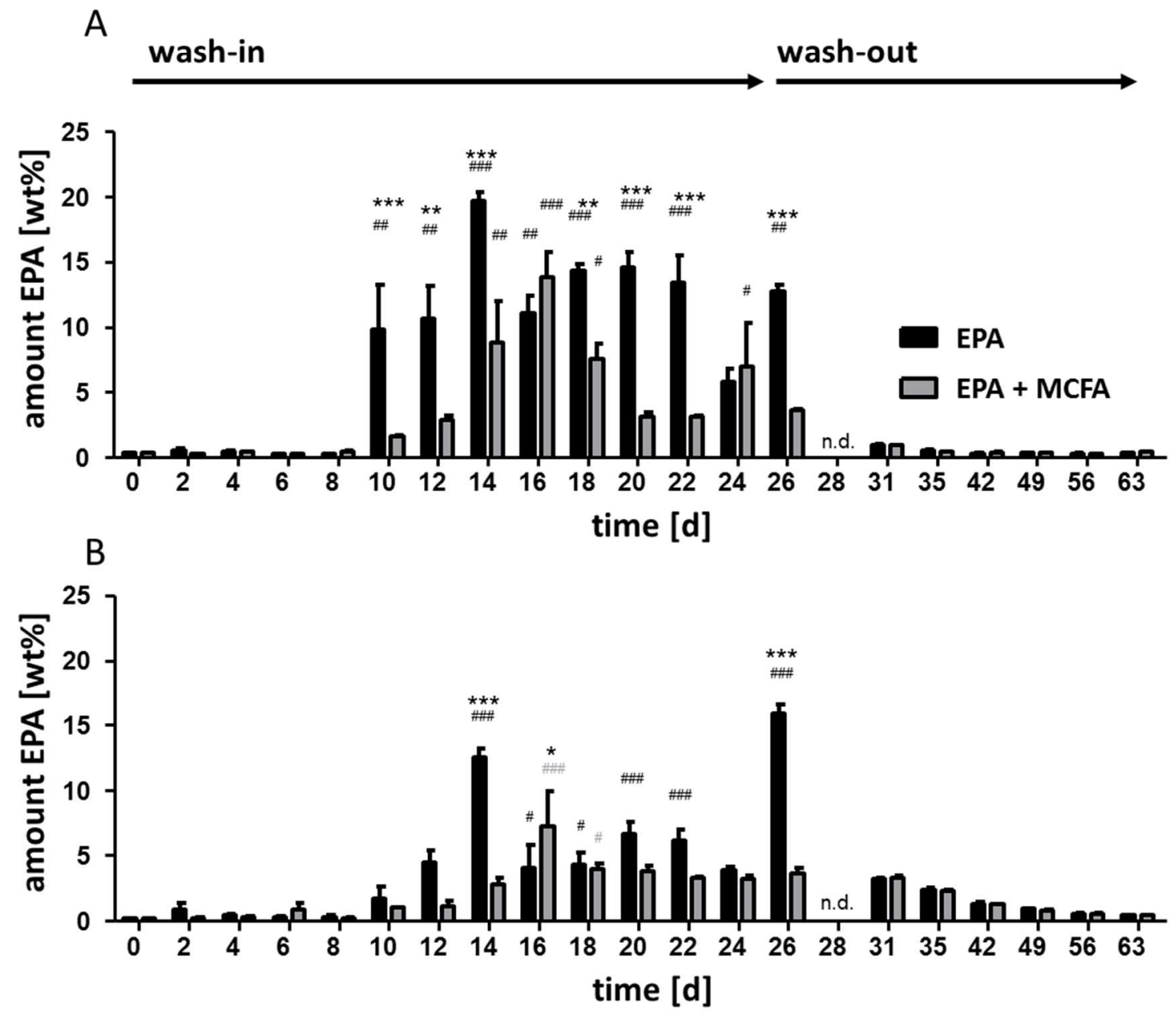
Figure 2. EPA incorporated into lung cells

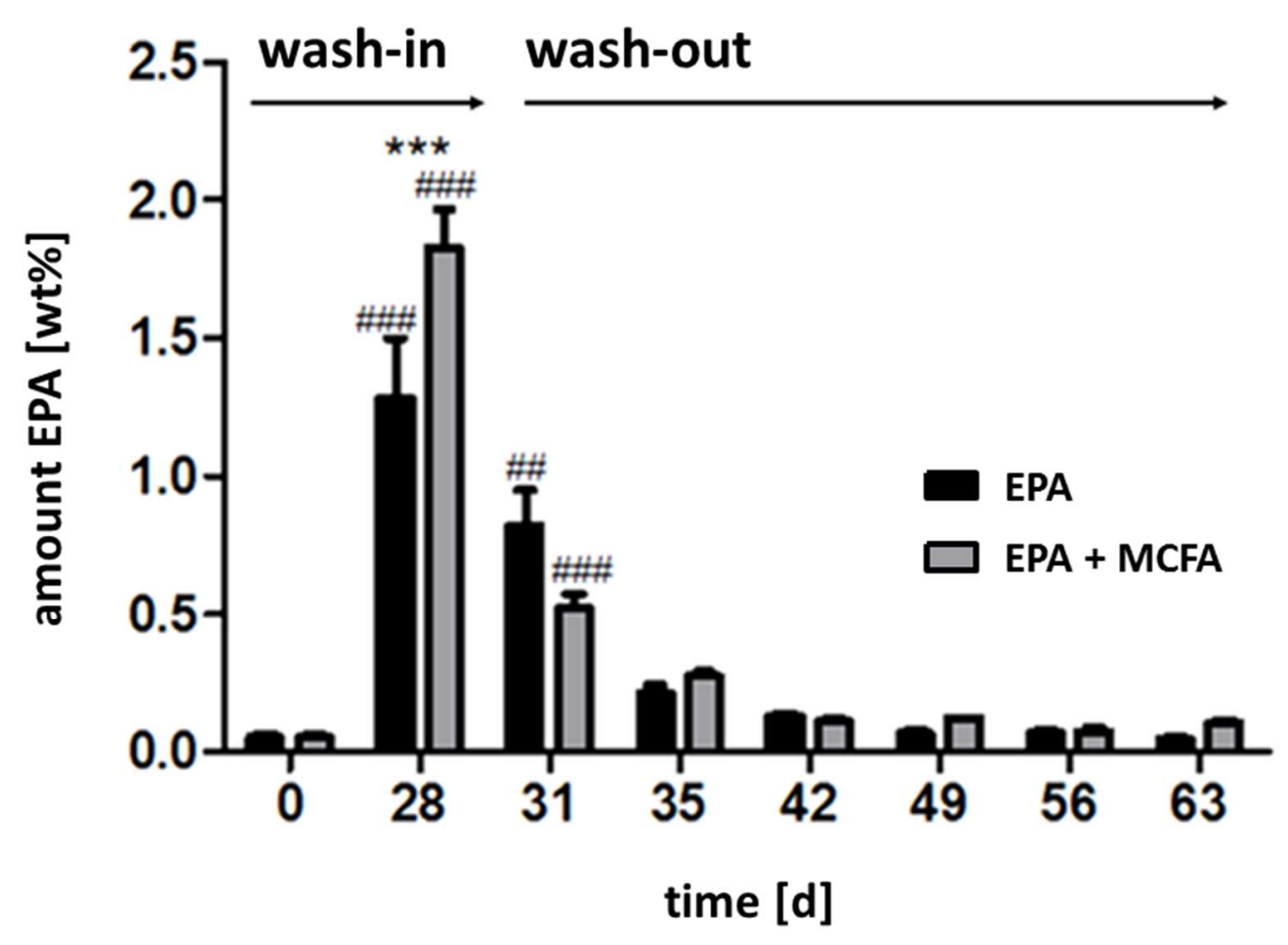


Figures

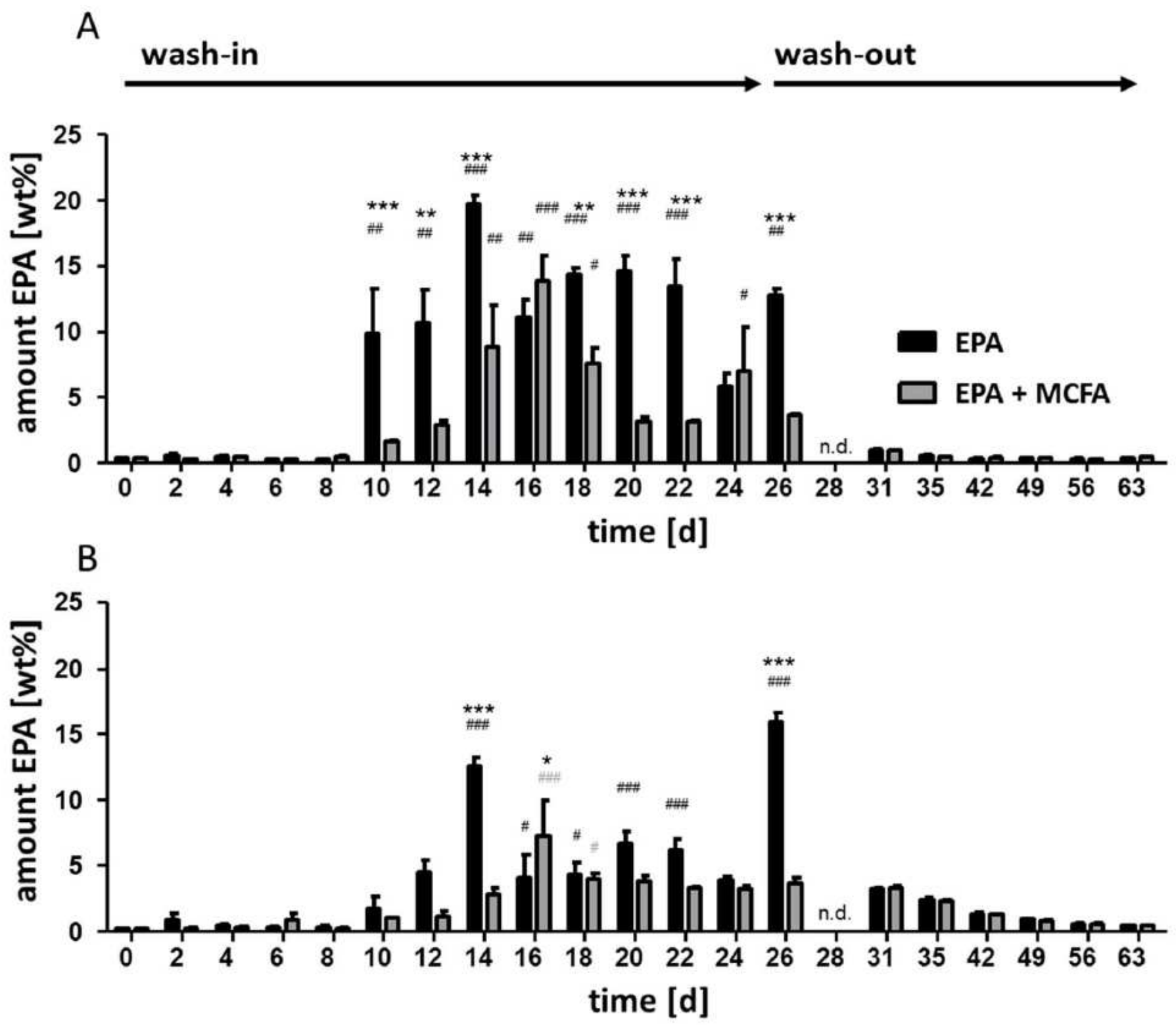

Figure 1

EPA concentration in blood 


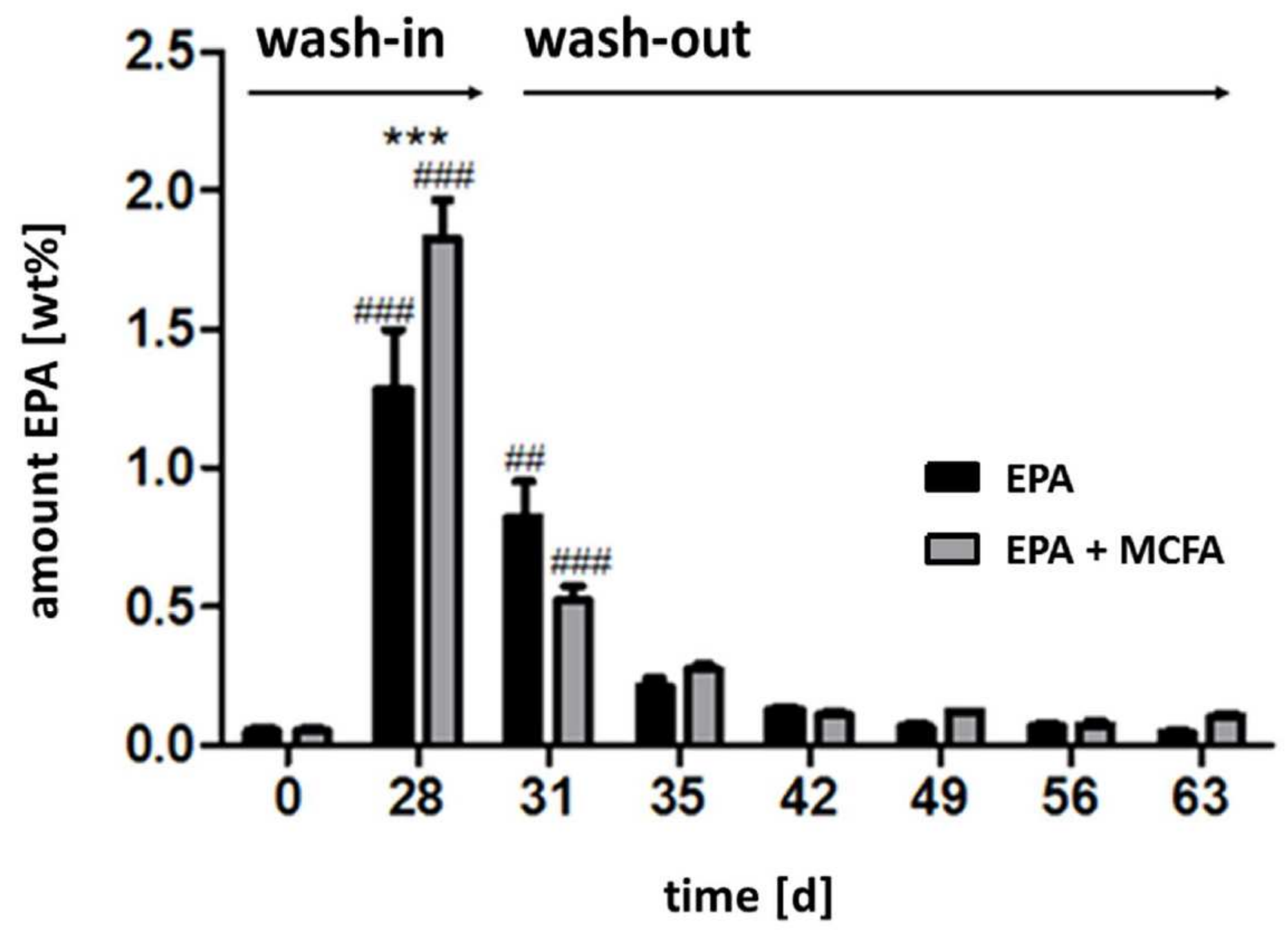

Figure 2

EPA incorporated into lung cells 